\title{
A New Camera System for Special Jobs in Terrestrial Photogrammetry
}

\author{
by Günther Voss.*
}

Though the principal and application of photogrammetry continues to be topographical surveys, and accordingly aerial surveying is internationally regarded to be of major importance, terrestrial photogrammetry is constantly growing in scope. The advantages of photogrammetry as a technique of measurement are well known. The fact that the information concerning the object to be measured or the events associated with that object is taken from a photograph often makes photogrammetry the only method of measurement available. Even when other approaches are possible, the savings involved often weight the scales in favor of the survey photograph. This is especially the case when the object is hardly accessible for direct measurement or is altogether inaccessible or possesses a complicated shape. Another factor is the simultaneity of the information recording process. Another argument in favor of photogrammetry is the fact that the interval of time during which the information is procured, i.e. the taking of the photograph, is comparatively brief, and that the object need not be available later on for the plotting process. On the other hand, this sets certain requirements for taking the photograph and hence for the camera employed, since details that are not disclosed often can not be filled in later.

During the past ten years the Phototheodolite Photheo 19/1318 (Fig. 1) has often been utilized for surveying in special fields, although it was developed for topographical surveying. It usually handled these jobs very well. It was found, how-

* Dipl.-Ing., VEB Carl Zeiss JENA

Journal of the Japan Society of Photogrammetry; Vol.

7, No. 1, 1968. ever, that a system of that kind was far from the optimum solution for every case, requiring concessions in the matter of costs. The angle of view and the fixed focusing (normally set at infinity) made special demands upon the photographing procedure. If we assume the existence of camera systems of other kinds, we can assign the place it deserves to the $19 / 1318$ system, that is, wherever a comparatively long focal length combined with a large-sized picture is needed or is wanted.

A different camera system is now available. It is based on a wide-angle objective of the Lamegon type, which has been derived from existing aerial camera lenses. The outstanding properties of this objective are set forth below.

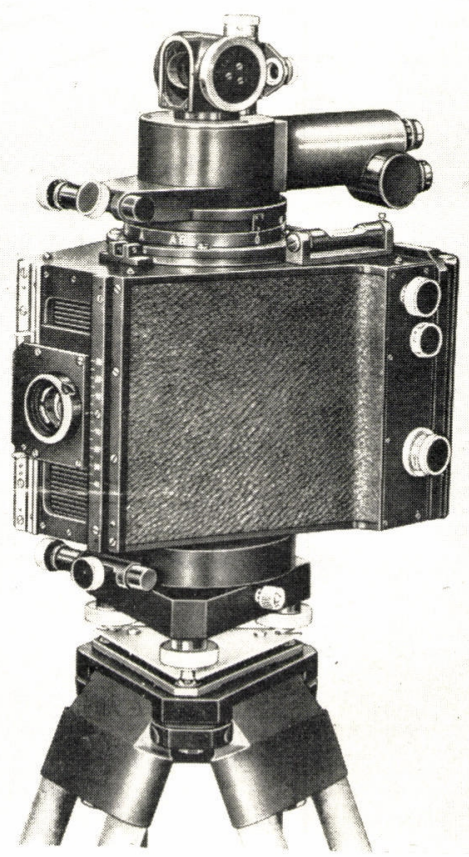

Fig. 1. Phototheodolite Photheo 19/1318 of VEB Carl Zeiss JENA 


\section{The Lamegon $5.6 / 55$}

The lens has a focal length of $56 \mathrm{~mm}$ and is designed for taking survey photographs $80 \mathrm{~mm} \times 80 \mathrm{~mm}$ in size. Its relative aperture is $\mathrm{f}: 5.6$.

\subsection{Geometric Properties}

Focal length and size of picture provide a $79 \mathrm{~g}$ angle of view, horizontally and vertically. The diagonal angle of view is $100 \mathrm{~g}$. This wide angle has been chosen deliberately. When photogrammetry is employed for special jobs, the problem often requires the photographing of as large an area as possible with a relatively short distance between camera and object. The short distances between camera and object are governed by the prevailing conditions for photography. The necessity of covering as large an object space as possible is largely dictated by economic considerations. As few stereo models as possible should be required to cover an object, so as to render subsequent plotting economical. On the other hand, the requirement that the information obtained be gathered simultaneously often means that the whole object has to be photographed in a single model. The same arguments apply to the surveying of events, which likewise have to be covered by a single pair of stereo photographs when only one camera is employed.

The wide angle of view involves correspondingly severe demands on the planarity of the photographic emulsion employed, of course, if the full size of the picture is to be utilized.

The lens has very low distortion. When focused for very long distance the distortion is no more than \pm 3 microns. The distortion curve is shown in Fig. 2 (solid line). The conditions prevailing when the lens is focused upon near objects and their effect upon the picture coordinate errors and parallax error will be dealt with in the course of the present article.

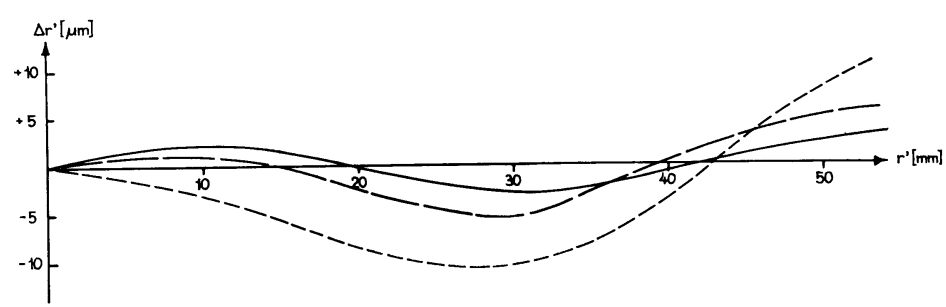

Fig. 2. Lamegon 5.6/55. Distortion curves (solid line: parfocalized at $\infty$; long dashes: parfocalized at 8 meters; short dashes: parfocalized at 4 meters)

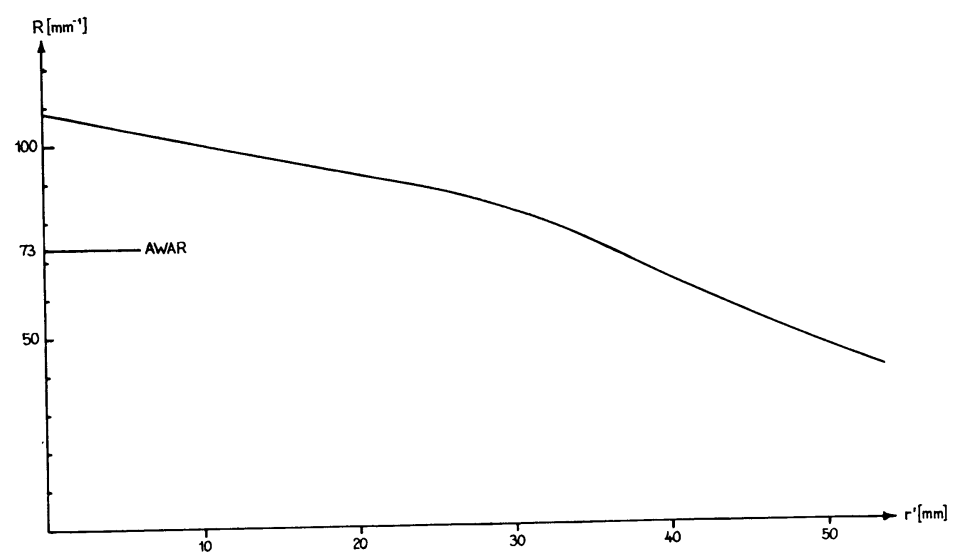

Fig. 3. Lamegon 5.5/55, Resolving power 


\subsection{Qualitative Properties}

In Fig. 3 resolving power is plotted versus distance from the center of the image. Values for a series of lenses are as follows: over 100 lines per $\mathrm{mm}$ at the center of the picture, over 60 lines per $\mathrm{mm}$ at the margin, and 40 lines per $\mathrm{mm}$ at the farthest corner. The area-weighted average (AWAR) is almost 75 lines per $\mathrm{mm}$. These are the results of high-contrast tests $(\log \mathrm{k}=2.0)$ with a high-resolution emulsion (ORWO-
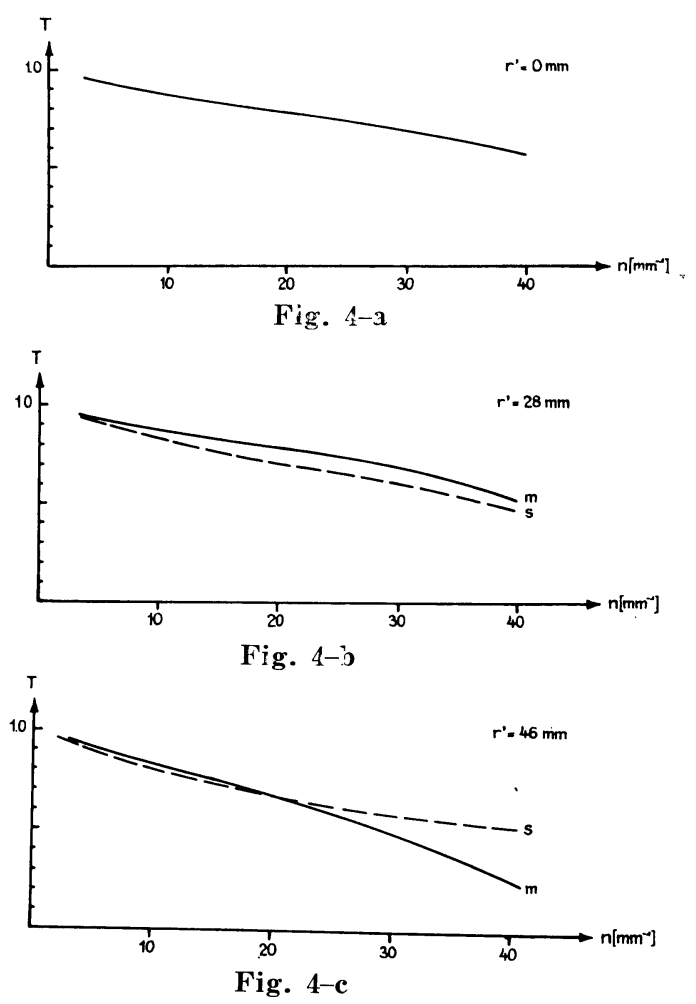

Fig. 4 a-c. Lamegon 5.6/55. Modulation transmission functions for three different distances from the center of the image
Topo).

The modulation transmission functions at three different distances from the center of the image are shown in Figs. 4a, 4b, 4c. The curves are based on the utilization of a panchromatic emulsion.

Lastly, Fig. 5 shows the brightness distribution in the plane of the image, based on the center of the image.

\section{The Stereometric Camera SMK $5.5 / 0808$}

These geometric and qualitative properties of the Lamegon 5.6/55 lens provide an excellent basis for utilizing the lens in a close-up camera. The Lamegon was therefore employed in designing the new Stereometric Camera SMK 5.5/0808.

\subsection{Principal Problems}

As we know, the principle underlying a stereometric camera is the combination of two cameras rigidly attached to each other, photography being done as in the normal case of photogrammetry. Both camera axes are perpendicular to the line joining the two projection centers (base) and parallel to each other. Ordinarily, the base and the camera axes are horizontal when taking the pictures. Departures from this standard position are usually feasible from the design standpoint, though the "normal case" should always be available. This is a decisive advantage. The job of relative orientation of the two camera positions, which is unpleasant and time-consuming, especially for someone who is not a professional photogrammeter, is eliminated. Ab-

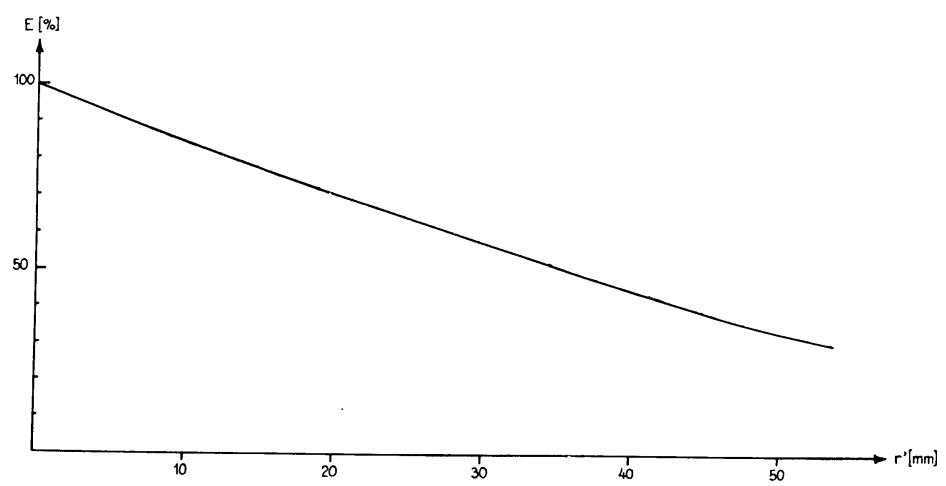

Fig. 5. Lamegon 5.6/55. Distribution of light in the image plane 
solute orientation is the only alignment of the camera that is required. This, in turn, does not affect the geometric conditions of the resultant model. The only consequence are transformational relations between the coordinate systems of the object and model, and these latter are of minor significance compared to the inaccuracies of the system. For example, planimetric plotting of a pair of photographs taken in the standard position mentioned above does not make too high demands of accuracy in the leveling of the system.

On the other hand, confinement to a fixed base entails certain disadvantages in the setup for taking the photographs. As we know, the depth error dY (and projectively, $\mathrm{dX}$ and $\mathrm{dZ}$ as well) produced by the parallax error $\mathrm{dp}$ increases as the square of the definition in depth, Y:

$$
d Y=-\frac{Y^{2}}{b \cdot c_{k}} \cdot d p
$$

It may be compensated to some extent by choosing the right base length $b$. On the other hand, approaching the object too closely (with a fixed base) or using too high a magnification of the base renders stereoscopic viewing and hence faultless plotting impossible. The limit depends on the structure of the object; in most cases this limit is reached when the base ratio $\vartheta=b / Y=1 / 3$.

In order to provide a certain variation of applications, the Stereometric Camera SMK 5.5/0808 is made in two base lengths, $400 \mathrm{~mm}$ and $1200 \mathrm{~mm}$. Figure 6 shows the resultant error dY for both systems due to a parallax error of $0.01 \mathrm{~mm}$.

A rough idea of the feasible camerato-subject distances for various accuracy requirements may be obtained from this diagram. One would not go wrong by setting the limit of plottability as 20 to 25 times the base lentgh.

In view of the camera-to-subject distances to be anticipated, the fixed parfocalizing distances have been set at $8 \mathrm{~m}$ for the $1200-\mathrm{mm}$ base and $4 \mathrm{~m}$ for the $400-\mathrm{mm}$ base.

On this basis, the lack of picture definition for object points beyond these distances may be calculated from the following formula:

$$
d^{\prime}=\frac{f^{2}}{d} \frac{\left|Y-Y_{0}\right|}{Y \cdot Y_{0}}
$$

where $d^{\prime}$ is the diameter of the circle of confusion, and $d$ is the f-stop number. These curves are shown in Figs. $7 \mathrm{a}$ and $7 \mathrm{~b}$. In the Stereometric Camera 5.5/0808 the relative aperture of the objectives can be adjusted in the following range: $\mathrm{f}: 5.6$ to

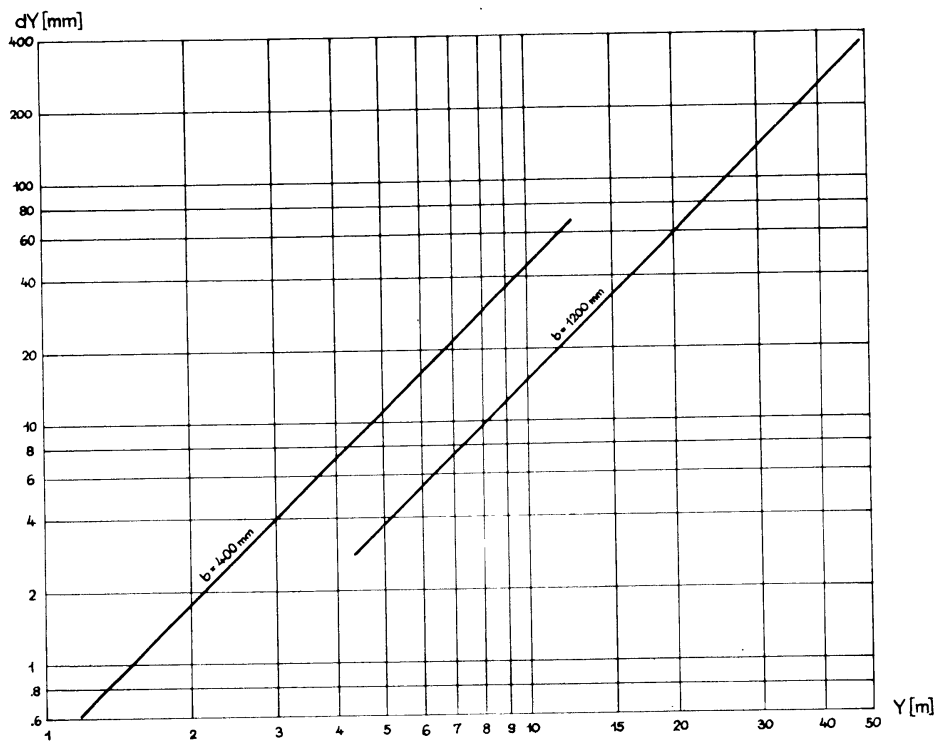

Fig. 6. Stereometric Camera SMK 5.6/0808. Depth error $d Y$ as a function of the object distance $Y$, based on a parallax error $d_{p}=0.01 \mathrm{~mm}$ 
f:22 by setting the shutter diaphragms.

The increases in the distance between the back lens and the image required for the camera-to-subject distances noted above produce a change in photogrammetric dis-
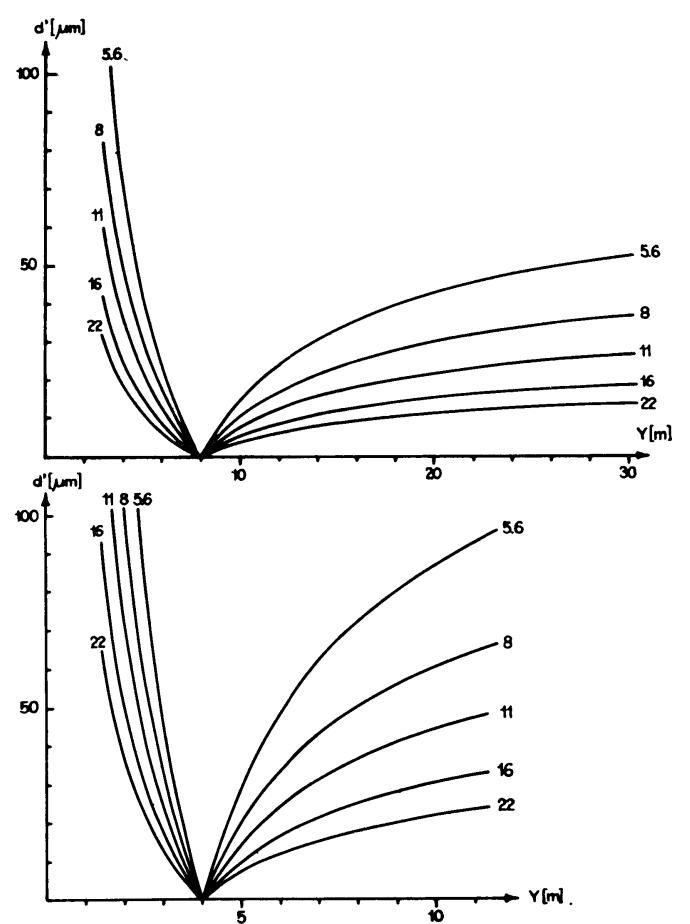

Fig. 7 a-b. Lamegon 5.6/55. Lack of picture definition as a function of object distance and relative aperture, for parfocalizations at 8 and 4 meters

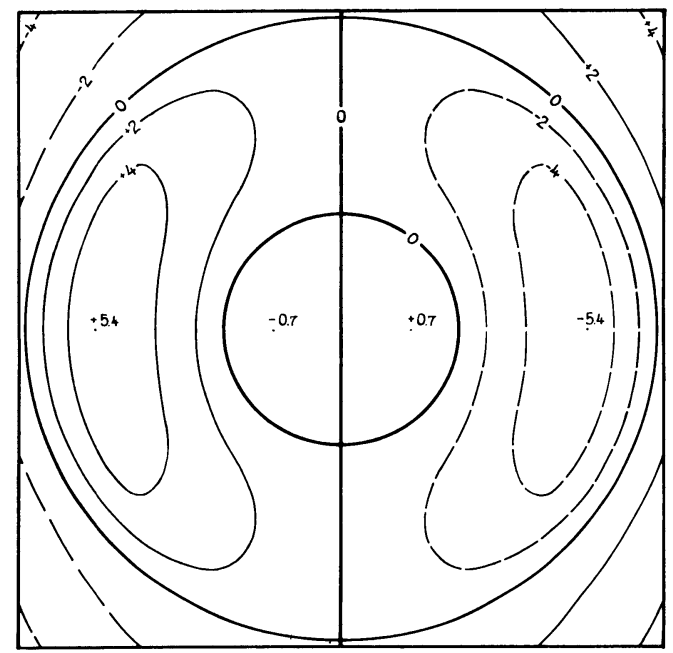

Fig. 8. Stereometric Camera SMK 5.5/0808, parfocalized at 8 meters $(1200 \mathrm{~mm}$ base $)$. Error of photograph coordinates, $d x$, based on rotation-symmetric radial distortion tortion. It might be mentioned in passing that the camera constants are all chosen so that the maximum distortion is the same over the entire image field. Corresponding curves, plotted for series-produced lenses, are shown in Fig. 2 (broken lines).

It is of interest to determine the effect of distortion upon photograph coordinates and parallax in the special case. Figures 8 and 9 show the photograph coordinate er-

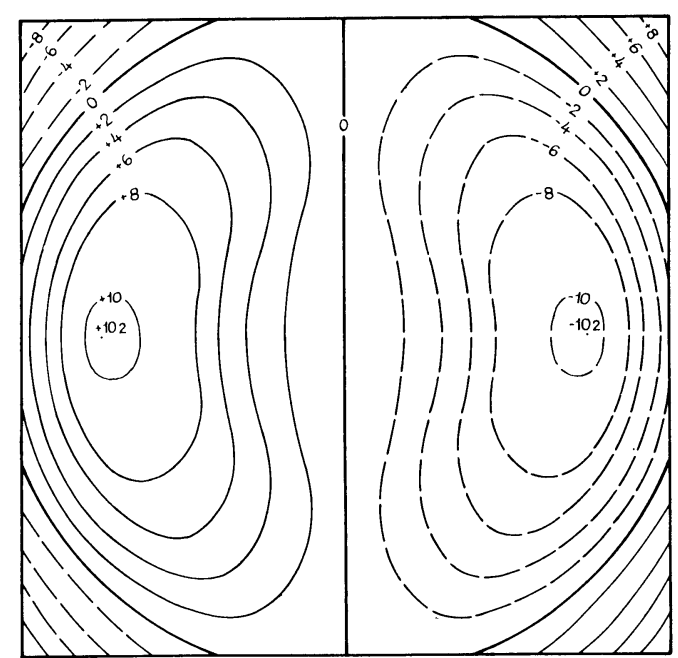

Fig. 9. Stereometric camera SMK 5.5/0808, parfocalized at 4 meters ( $400 \mathrm{~mm}$ base). Error of photograph coordinates, $d x$, based on rotation-symmetric radial distortion

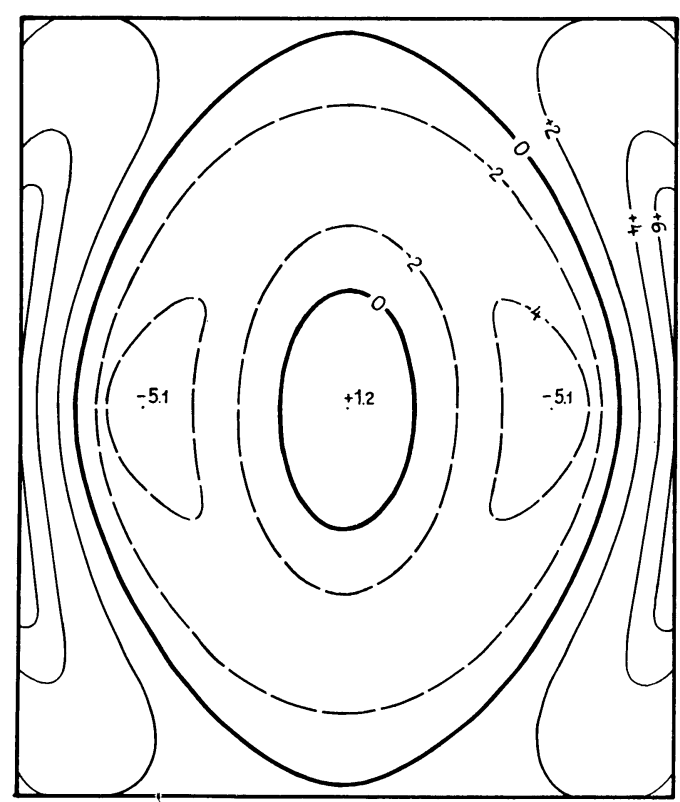

Fig. 10-a 
rors in $x^{\prime}$ for the distortions shown in Fig. 2 and parfocalization to 8 and 4 meters. The errors in $z^{\prime}$ are found by rotating the figures through $100 \mathrm{~g}$.

Due to the small parallaxes generally prevailing in terrestrial photogrammetry, when the curves are at all continuous the distortion is converted into parallax errors, which are normally smaller than the maximum distortion. The parallax errors for the $1200-\mathrm{mm}$ base (parfocalization to 8

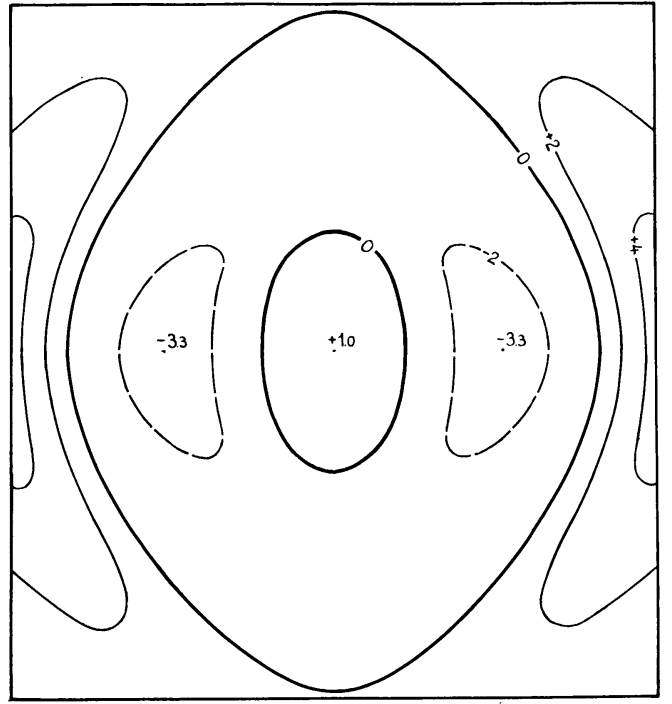

Fig. 10-b

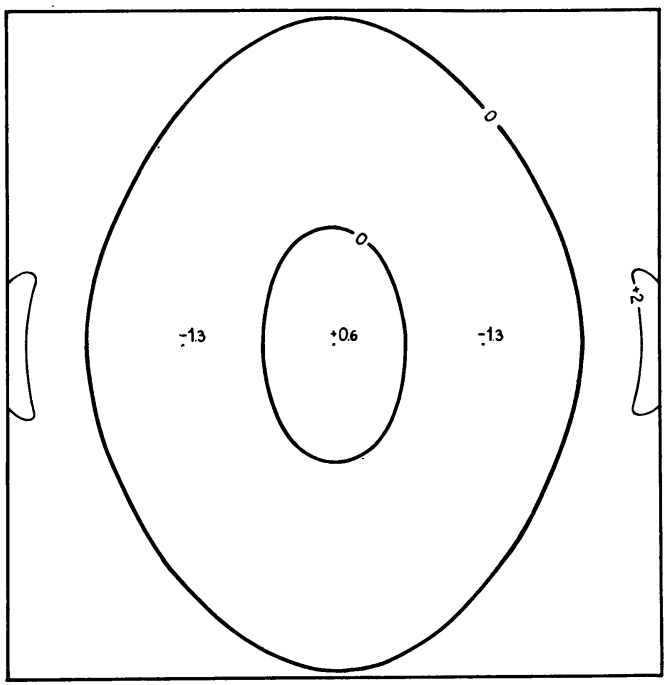

Fig. 10-c

Fig. 10 a-c. Stereometric Camera SMK 5.5/ 0808, parfocalized at 8 meters $(1200 \mathrm{~mm}$ base $)$. Parallax error, $d_{p}$, based on rotation-symmetric radial distortion meters) are shown in Figs. 10a to 10c for three different object distances. Similarly, the lines of equal parallax error for the 400-mm base, again for three object distances, are given in Figs. 11a to 11c. The maximum values lie at the horizon trace or at the extreme lateral limits of the model. Even for the greatest parallaxes they lie well within the orders of magnitude demanded. With increasing object distances, the effect upon the photograph co-

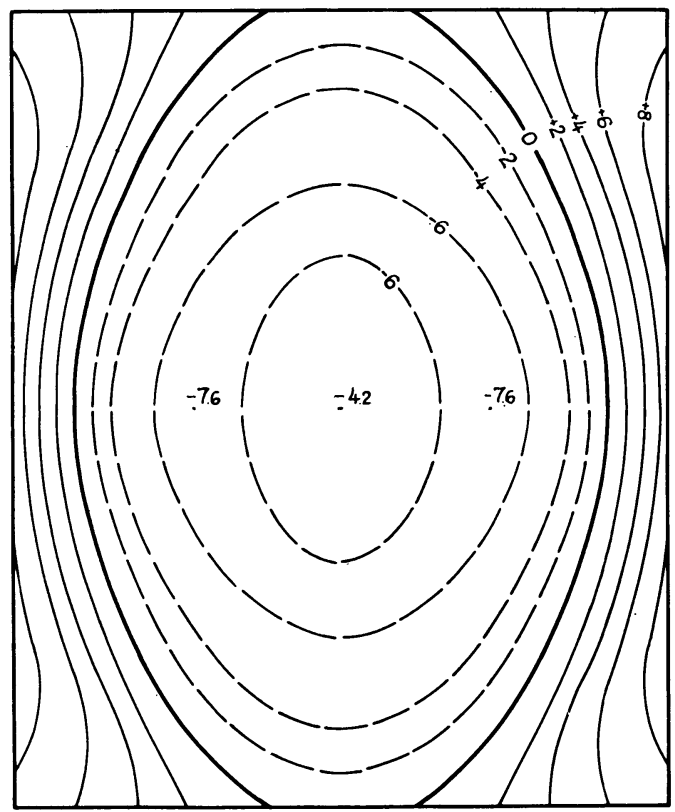

Fig. 11-a

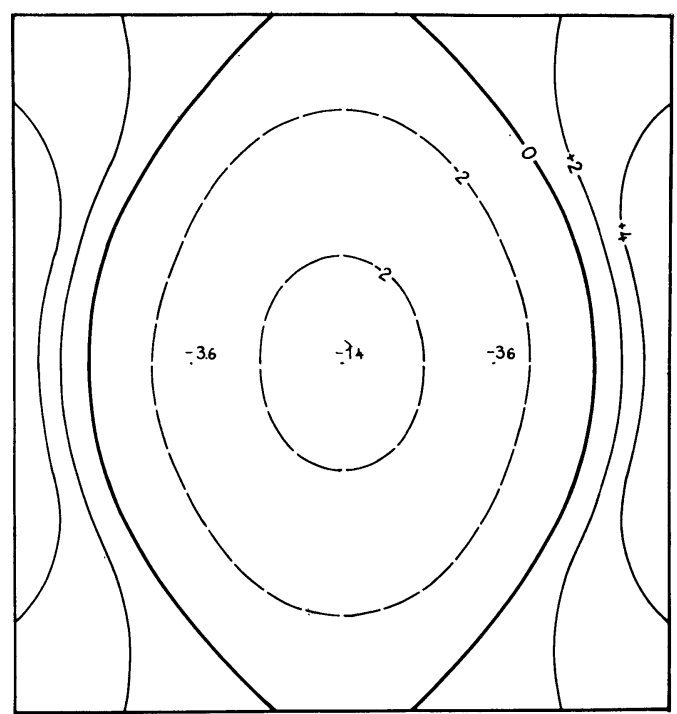

Fig. 11-b 
ordinates that is independent of distance predominates.

\subsection{Description of the Instrument}

Special weight was attached to two considerations when designing the Stereometric Camera 5.5/0808. It was stated above that alignment for the normal case was a basic requirement for stereo cameras. In view of the fact that defective relative orientation entails extremely uneconomical fitting during the subsequent plotting, the stability of the design must meet extremely severe

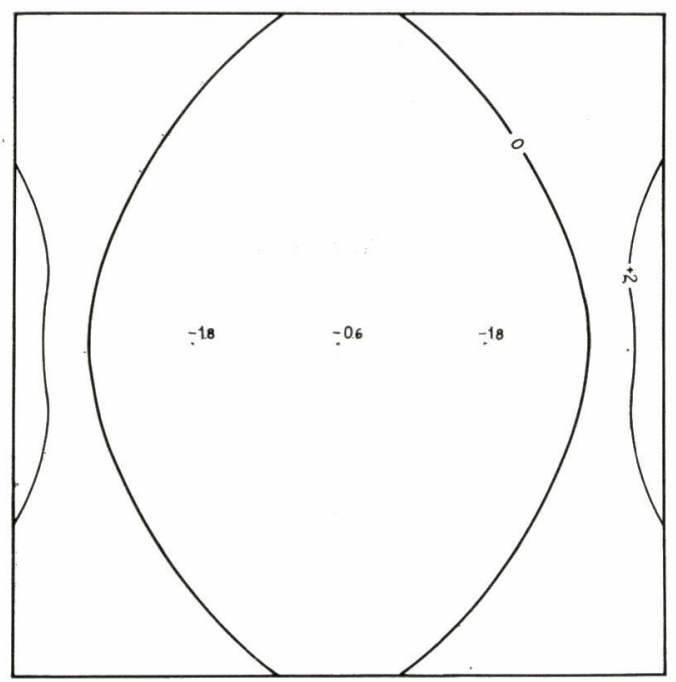

Fig. 11-c

Fig. 11 a-c. Stereometric Camera SMK 5.5/ 0808, parfocalized at 4 meters $(400 \mathrm{~mm}$ base) . Parallax error, $d_{p}$, based on rotation-symmetric radial distortion standards. Compare the diagram shown in Fig. 6, which is based on a parallax error of no more than 10 microns. Moreover, an endeavor must be made to do without pass points for photography, so as not to have to waste the time saved by having to make laborious ground surveys for applications made when time is short. Thus, the user must be able to rely on the camera yielding pairs of photographs that are practically rigorous examples of the normal case. The high stability of the instrument entails a slight increase in weight.

The second consideration is operation that is foolproof and as simple as possible, especially as the operating personnel may well be persons without photogrammetry training.

That is why all the operating controls and monitoring elements are arranged centrally on the instrument (Fig. 13).

The operating controls act upon both cameras simultaneously. They comprise:

Setting the shutter speeds: B, 1 to $1 / 500$ th sec.

Setting the stops: f:5.6 to f:22.

Winding up the shutter.

The electromagnetic shutter release.

Setting the illumination of the fiducial marks and the auxiliary data recorded on the photograph.

Setting the counter to zero.

The counter, with digits from 0 to 9 ,

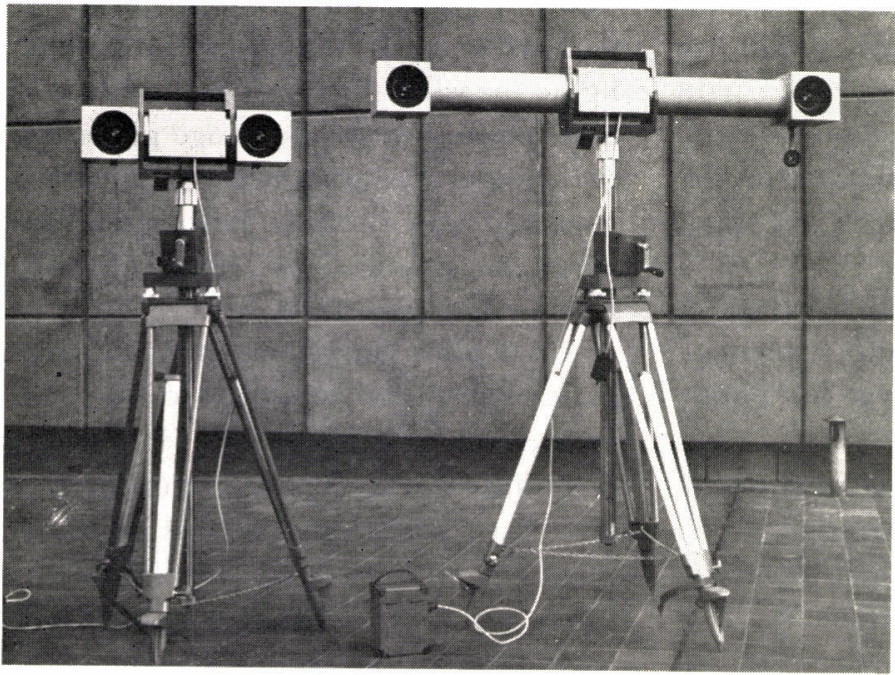

Fig. 12. Stereometric Camera SMK 5.5/0808, with base lengths of $400 \mathrm{~mm}$ and 1200 mm of VEB Carl Zeiss JENA 


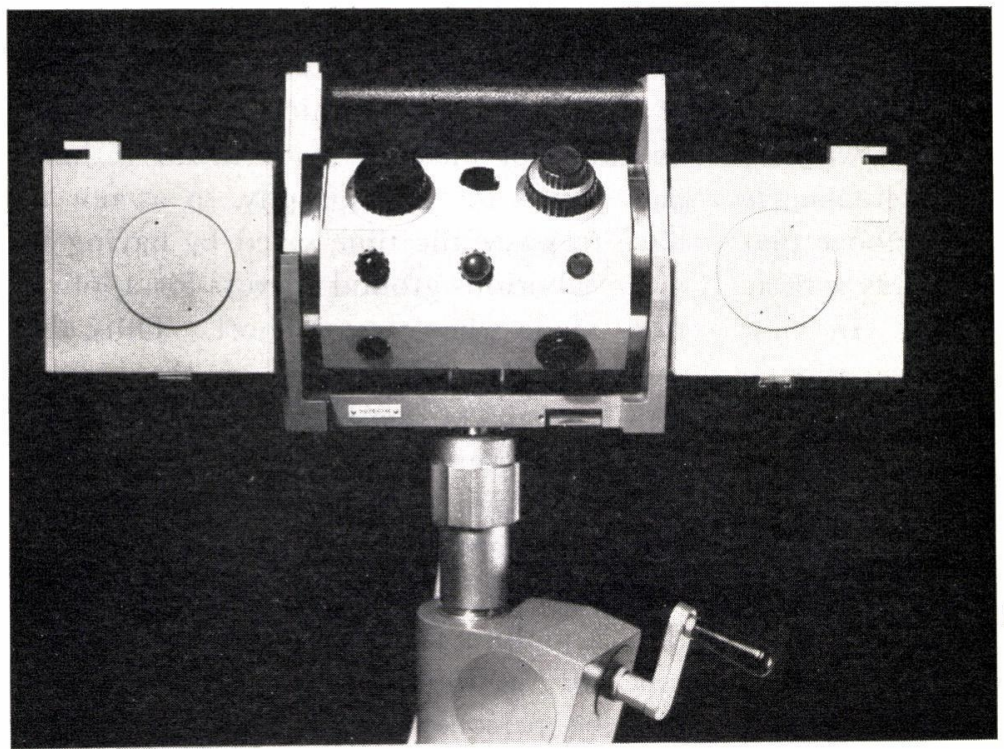

Fig. 13. Stereometric Camera SMK 5.5/0808. Central control panel

advances one unit each time the shutter is cocked; the number appears in both photographs of a stereo pair.

The electromagnetic shutter release provides a high degree of synchronization of the shutter openings, if desired. Connection of a flash unit (to the shutter of the left camera) is important for photographs taken at night or in poor light.

The electrical illumination of the fiducial marks makes them completely independent of the distribution of brightness over the object. These marks are outside the frame of the picture and comprise a dot and a concentric circle around it. They are adjusted so that the central point of the image defined by them coincides with the principal point of the image. Light for the marks is supplied from a central light source for each camera through a lightconducting cable. The auxiliary data are transmitted to the emulsion in the same way. These data include:

The camera constant for each camera

The count number for both cameras

The base length, shown in the left photograph

The serial number of the instrument, shown in the right photograph

The writing space of a slide-in note pad is also photographed in both cameras. The note pads are used to record other data for the photograph, such as the date, the time, etc. The illumination time (the time during which the incandescent lamps are lit) remains constant. The illumination is adapted to the sensitivity of the emulsion by 4 -step series resistors. Illumination is effected automatically by a special electrical circuit connected to the shutter release.

The control panel also contains the monitoring elements, in the shape of pilot lamps of different colors.

A white lamp indicates that the circuit for illuminating the fiducial marks is live, after the selector switch has been actuated. At the same time, this lamp illuminates the circular spirit level for absolute orientation. A green lamp indicates that the apparatus is ready to take photographs. It lights up when the emulsion surfaces have been positioned in the image planes by the plateholder pressure devices in both cameras and the shutters have been cocked. Once the shutters have been released, the green pilot lamp goes out.

A red pilot lamp then indicates that the plates have been exposed, so that the apparatus is not ready for further photographing. No mechanical interlock of shutter release is provided at this stage. After recocking the shutters (the green lamp lights up again), the photographs can be taken again, though the danger of faulty 


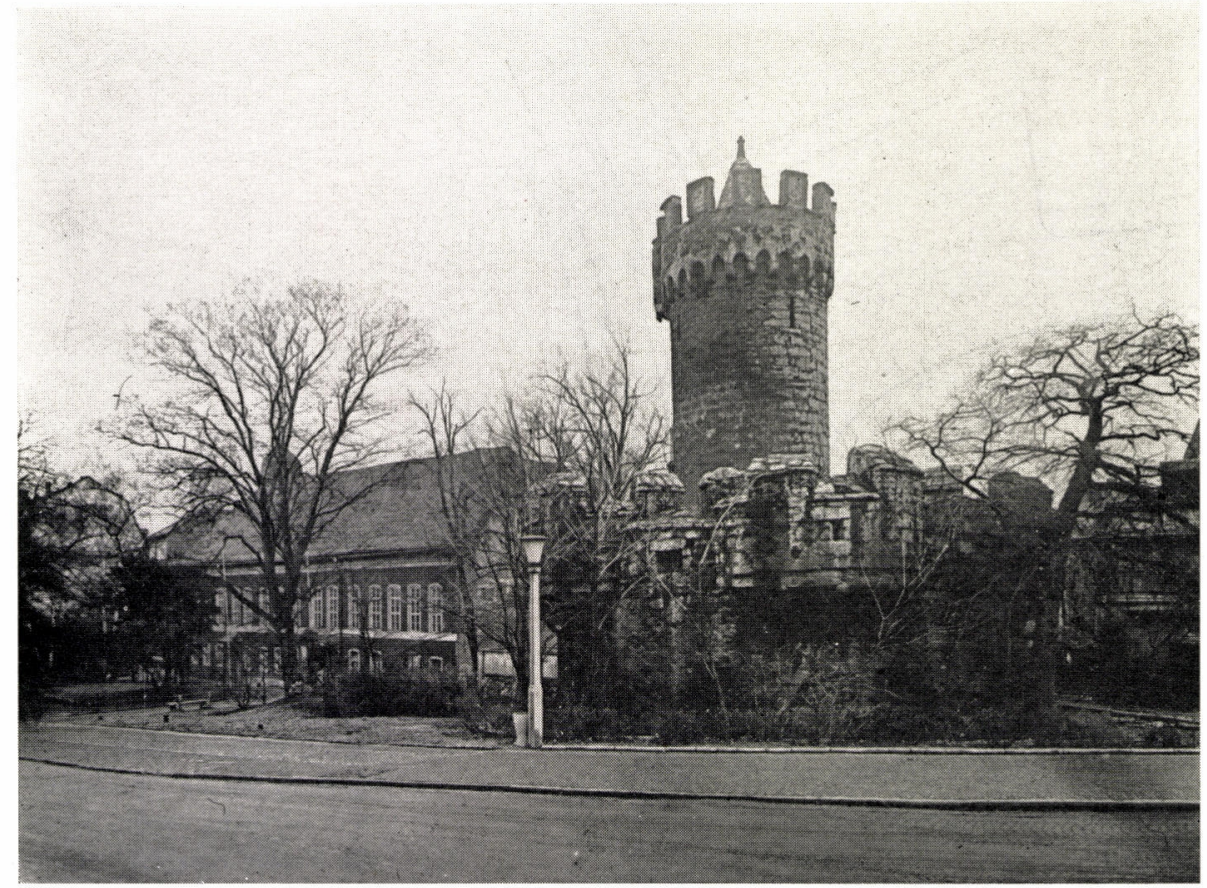

Fig. 14. Stereometric Camera SMK 5.5/0808. Photogram

operation (double exposure) is eliminated by the fact that the red warning lamp does not go out. The red pilot lamp likewise serves as a check on the light sources. If the incandescent lamps are intact, it lights up when the selector switch has been thrown to the proper position, though the incandescent lamps do not light up then.

When this ingenious arrangement of optical indicators is properly employed, it prevents faulty operation of the apparatus.

Another safety feature involves the insertion and withdrawals of the plateholders. The slides covering the insertion slits on the sides of the camera bodies are so designed that they can be opened or closed only when the plateholders are not in position. This prevents damage to the fiducial mark pins or the plane glass plates, surface-ground with the greatest precision, which fix the image plane.

The glass pressure plates are cleaned after the rear covers of the camera bodies are unscrewed. Realization of the image plane by means of a plane glass surface makes it possible to employ flat film as the emulsion base, this being particularly im-

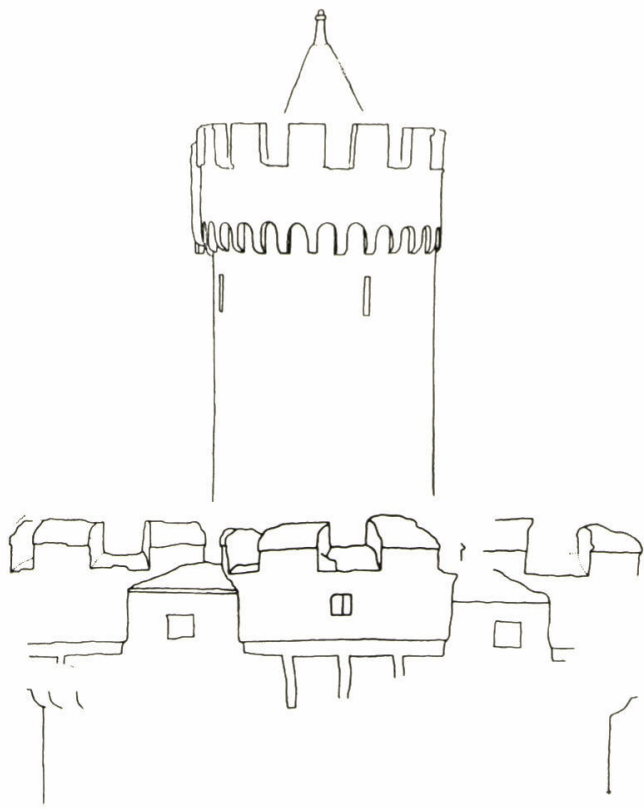

Fig. 15. Plotting a pair of stereo photographs taken with the Stereometric Camera SMK 5.5/0808: elevation of an architectural subject

portant when color photographs are to be taken.

Photographic glass plates in the standard size of $9 \mathrm{~cm} \times 12 \mathrm{~cm}$ are the normal material employed. The plateholders are the 


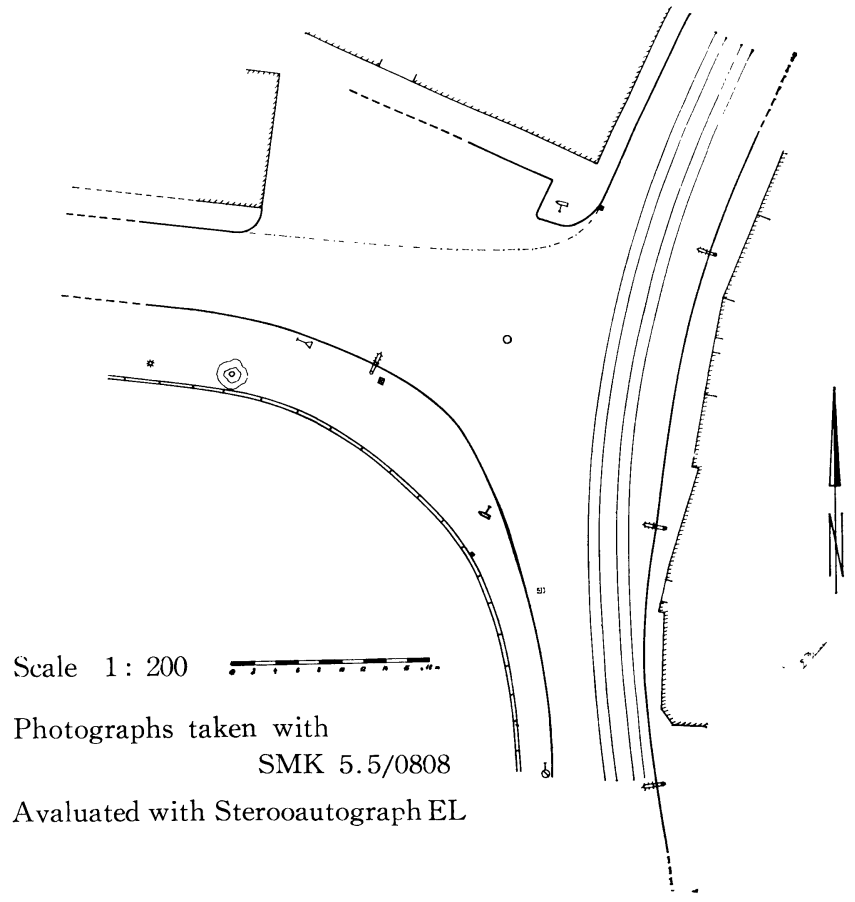

Fig. 16 Plotting a pair of stereo photographs taken with the Stereometric Camera SMK 5.5/0808: preliminary survey of a street crossing (site of many traffic accidents) for the Traffic Spuad of the police ordinary commercial type.

In the normal model of the Stereometric Camera SMK 5.5/0808, two operating positions of the camera axes are provided, horizontal and vertical, with the horizontal position of the base remaining unchanged. The two spirit levels required for exterior orientation can be conveniently viewed in a folding mirror.

The cameras operate on a d.c. voltage of 12 volts and are thus readily connected to the storage battery of the transporting vehicle.

The tripod supports a tribrach with a vertical spindle, which enables the cameras to be raised or lowered by means of a hand crank according to the requirements of the job. Their maximum height above the ground is 2.75 meters. The tripod head is fitted with another spirit level for preliminary leveling.

The equipment is also provided with an optical finder, which can either be attached to the cameras as an orientation aid or employed as a hand-held instrument for selecting the camera station.

\subsection{Applications}

The field of application of the Stereo- metric Camera 5.5/0808 is effectively specified by the relationships between the space coordinate error and the camera-to-subject distance, shown in Fig. 6. It is customary to list the respective fields of application. In view of the fact that photogrammetry is being employed in more and more "special fields", that there no doubt are possible applications as yet unspecified, and that, despite the individuality of these fields of application, the possibility of securing sufficient information about the object depends in the last analysis upon the instrumentation, a listing of that sort becomes superfluous.

What is necessary, rather, is to provide the specialist in a discipline for which photogrammetric surveying is appropriate with the necessary pointers concerning the given possibilities of application. That is why the present article goes beyond the mere description of the instrument.

As indicated above, these possible applications are manifold, extending far beyond such common fields as the photographing of traffic accidents or architectural surveys. We trust that the present article will furnish further stimuli along these lines. 